\title{
A systematic fitting procedure for accurate force field models to reproduce ab initio phonon spectra of nanostructures
}

\author{
Christopher Barrett ${ }^{1,2}$ and Lin-Wang Wang ${ }^{2 *}$ \\ ${ }^{1}$ Department of Materials Science and Engineering, University of California, Berkeley, California \\ 94720, USA
}

2Material Sciences Division, Lawrence Berkeley National Laboratory, Berkeley, California 94720, USA

*lwwang@lbl.gov

\begin{abstract}
A fitting procedure is presented to use a valence force field model to generate the phonon spectrum of large nanostructures. This approach uses a relatively large number of parameters ( 50$)$ in order to generate the accurate $a b$ initio phonon spectrum. Since the emphasis is in the accuracy rather than the transferability, it can only be used in similar bonding environments. Because of this, a reliable and automatic fitting procedure is essential. We discuss the detailed aspects of the fitting procedure, including the stages of fitting, the type of $a b$ initio values used for the fitting, the weighting factors for different quantities, the number of ab initio data points needed, as well as the uniqueness of the parameters. We found that the parameters cannot be determined uniquely, indicating interdependence of the parameters. Nevertheless, the different parameters resulted from different fits all give accurate phonon spectrum compared to ab initio results. We have used the fitted valence force field model to study the phonon spectra of CdSe nanowires.
\end{abstract}

Keywords: Classical Force Field Model; Fitting; Phonon Spectrum; Polar Semiconductor; Nanostructure; Surface

Abbreviations: The abbreviation "DCM" is used to refer to "double charge model" in this paper, as discussed in Section 2 and described in Ref. 30.

\section{Introduction}

Classical force field Hamiltonians, which represent the total energy of a given system in terms of atom-atom pair interactions [1-5], three-body interactions, embedded-charge energies [6,7], and bondangles energies [8], have been one of the main approaches for material science simulations. This includes the Lennard-Jones pair potential model for ionic systems [2], the Tersoff three-body potential for systems like Si [9], the embedded atom model for metallic systems [10], the valence force field (VFF) 
model for all the covalent bond systems (semiconductors [11], organic systems [12-17], and biological systems [18]), as well as the recent reactive force field [19]. Although the ab initio quantum mechanics simulations have gained tremendous popularities in recent years due to the increase of their speeds, some classes of problems are still better handled by classical force fields. These include systems with millions of atoms, or molecular dynamics simulations that span hundreds of nanoseconds. While immensely useful, the classical force field methods often suffer for their accuracy and reliability. One major issue is the number of parameters in a force field model. On the one hand, more parameters can make a force field fitting more accurate for a given data set, hence increase its accuracy. On the other hand, more parameters might potentially make the Hamiltonian less transferable to new situations which are not fitted. Historically, when a force field was developed, there might not be many data points to use in fitting its parameters. The available data points, especially the experimental ones, are usually rather scarce. Combined with the concerns for transferability, many force fields choose to have as few parameters as possible. This makes them transferable, but usually less accurate, e.g., when compared with ab initio results.

With the availability of ab initio calculations, the force field can be developed by fitting their parameters to $a b$ initio calculated results. Many modern force fields are developed this way, including CFF99 [20] and MMFF [21]. Nevertheless, even for these force fields, the transferability is a high priority when fitting their parameters. Part of the reason is that the force field is intended to be used for different systems without any refitting. Because of this, their accuracies can still be limited. Typically, for these force fields, the values of one parameter might only depend on the atom types related to the corresponding energy term (e.g., bond energy, or bond angle energy).

With the advance of computer speed and the lower cost of conducting ab initio calculations, different paradigms for this parameter fitting problem have appeared. In the extreme case, one can use many parameters (e.g., each pair interaction term with its own parameter), and fit those parameters on the fly during a simulation (e.g., to fit all the ab initio atomic forces at each step during a molecular dynamics simulation) [22]. In such an approach, the ab initio atomic force can be fitted exactly, but the fitted parameters can probably only be used near that particular atomic configuration.

Here, we are not interested in such an extreme case as for the on-the-fly fitting. We are interested in some intermediate situations where many parameters are used, and can be fitted automatically. The resulting classical formula is rather accurate in reproducing the ab initio results, but perhaps only applicable to a limited class of problems (the transferability is limited). For a different set of problems, the ab initio data should be regenerated, and the automatic fitting procedure should be reapplied to get the corresponding parameters. This approach has been used successfully for the cluster expansion method to describe the alloy energy [23]. In that method, typically 30 to 50 random lattice atomic configurations are generated, and ab initio energies of them are calculated. These energies are used to fit the energy parameters of different cluster expansion terms. The resulting cluster expansion Hamiltonian is then used to simulate the thermodynamic properties of the alloy. A similar approach has been used to model the nonharmonic interaction terms in a bulk system, with the third order interaction term parameters fitted to ab initio energies of randomly displaced atomic configurations 
from a perfect crystal [24]. In all these approaches, the automatic fitting procedure is the key point for the success of the whole procedure.

In this work, we will use the valence force field (VFF) model to calculate the phonon spectrum of semiconductor nanostructures (slabs, nanowires and quantum dots). One important criterion is to yield the surface-related phonon modes. Such phonon modes are important when studying the carrier dynamics in these systems. They can also be important for problems related to the infrared or Raman spectrum of attached molecules. While the existing VFF potentials (with their small number of parameters) might be useful in determining the atomic positions for a given nanostructure (e.g., to describe the atomic relaxation in a nanostructure heterojunction due to lattice mismatch), they are usually rather inaccurate in describing the phonon spectrum. The phonon spectrum is related to the second derivative of the total energy with respect to the atomic coordinates R. A good ground state does not guarantee good second-order derivatives. Figure 1 shows the phonon dispersion for bulk WZ CdSe using an existing VFF model, which is fitted to experimental bulk strain moduli, used for structural relaxation in Zinc-Blende and Wurtzite systems [25]. Figure 1 also compares it with the DFT phonon dispersion as well as that produced by the technique presented in this paper. We can see that there are major differences between the former two, with the existing VFF optical bands not even resembling those from DFT and the acoustic bands all being too high in energy. By contrast, the model presented in this paper resembles the DFT results much better quantitatively.

To increase the accuracy, we have significantly increased the number of parameters. This is particularly true for situations involving surfaces. Following the spirit discussed above, different surfaces can have their own corresponding set of surface parameters. In our case of wurtzite CdSe, for the bulk system, there are 21 parameters, while for the (1010) surface, there are an additional 53 parameters. The purpose of this paper is to (a) investigate the feasibility of this new approach, for its accuracy and transferability, and (b) detail the parameter fitting procedure so an automatic fitting will be possible. In the literature, the details of the fitting procedure are often not reported. This makes it difficult to reproduce the reported results. We want to address this issue in the current paper, meanwhile answering the following questions encountered during the fitting process: (1) How accurately can one reproduce the ab initio phonon spectra for a nanosystem? (2) What is the proper fitting procedure? Should we fit all of the system at once (e.g., bulk plus the surface), or fit different parameters in stages? (3) What ab initio data should be included in the fitting: energy, or force, or directly the phonon spectrum? (4) How should one automatically choose the weights in the fitting task function? (5) How many $a b$ initio data points are sufficient to ensure the quality of the fitting results? (6) Can the parameters be uniquely determined? Alternatively, is such a unique determination absolutely necessary? Overall, we will show that following our automatic procedure, it is possible to generate a VFF model which not only reproduces the bulk phonon band structures correctly, but also reproduces the surface-related modes correctly. This procedure provides a cheaper alternative to calculating the phonon spectrum of a nanosystem directly using ab initio methods, which requires $3 \mathrm{~N}$ self-consistent calculations, where $\mathrm{N}$ is the number of atoms in the system. We have used the resulting VFF Hamiltonian to study the phonon modes of nanowires, which are too large to be calculated by ab initio methods. 


\section{Model}

We will focus on an extension of the Valence Force Field (VFF) model originally developed by Keating [11], specifically a version of it that is now commonly used [26-29]. The bonding terms for this version of the VFF model can be written as:

$$
\begin{aligned}
& E_{b}=\frac{1}{2} \sum_{i} \sum_{j}^{n n(i)} \frac{3 \alpha_{i j}}{8\left(d_{i j}^{0}\right)^{2}}\left[R_{i j}^{2}-\left(d_{i j}^{0}\right)^{2}\right]^{2}+\frac{1}{2} \sum_{i} \sum_{j, k \neq j}^{n n(i)} \frac{3 \beta_{i, j k}}{8 d_{i j}^{0} d_{i k}^{0}}\left[R_{j i} \bullet R_{k i}-\cos \left(\theta_{i, j k}^{0}\right) d_{i j}^{0} d_{i k}^{0}\right]^{2} \\
& +\sum_{i} \sum_{j, k \neq j}^{n n(i)}\left\{\frac{3 \gamma_{i, j, k}}{8 d_{i j}^{0} d_{i k}^{0}}\left[R_{j i} \bullet R_{k i}-\cos \left(\theta_{i, j k}^{0}\right) d_{i j}^{0} d_{i k}^{0}\right]\left[R_{i j}^{2}-\left(d_{i j}^{0}\right)^{2}\right]+\frac{1}{2} \frac{3 \delta_{i, j, k}}{8 d_{i j}^{0} d_{i k}^{0}}\left[R_{i j}^{2}-\left(d_{i j}^{0}\right)^{2}\right]\left[R_{k i}^{2}-\left(d_{i k}^{0}\right)^{2}\right]\right\} \\
& +\frac{1}{2} \sum_{i} \sum_{j, k \neq j}^{n n(i)} \sum_{l \neq i}^{n n(k)} \frac{3 \varphi_{i, j, k}}{8 d_{i k}^{0} \sqrt{d_{i j}^{0} d_{k l}^{0}}}\left[R_{j i} \bullet R_{k i}-\cos \left(\theta_{i, j k}^{0}\right) d_{i j}^{0} d_{i k}^{0}\right]\left[R_{i k} \bullet R_{l k}-\cos \left(\theta_{k, i l}^{0}\right) d_{k i}^{0} d_{k l}^{0}\right]
\end{aligned}
$$

where $i$ refers to an atom, $j$ and $k$ refer to nearest neighbors of $i, l$ refers to a nearest neighbor of $k, R_{i}$ is the atomic position of atom $i, R_{i j}=R_{i}-R_{j}, d_{i j}^{0}$ refers to the ideal bond length of atom pair $i-j, \theta_{i, j k}^{0}$ refers to the ideal angle $j-i-k$, and $\alpha, \beta, \gamma, \delta$, and $\varphi$ are force parameters to be fitted. The five terms in Eq.(1) are bond harmonic, angle harmonic, bond-angle, bond-bond, and angle-angle interaction terms. Note, since we focus on bulk-derived systems instead of organic molecules, we have not included any torsion-angle terms.

In order to use such a model for polar semiconductor structures, in which we are interested, it is necessary to introduce an ionic charge model so that the full model can reproduce the dynamics of the Born Effective Charge. Simply applying the Born Effective Charge as an ionic charge, though, results in problems of structural collapse due to too-large electrostatic charges. In this work we will use the double charge model (DCM) [30], which can produce the correct Born Effective Charge and electrostatic charge. In this model, there are two point charges associated with each atom that do not exert force on each other: One is $q$ with its position at the atomic position $R$, another is $q^{\prime}$ with its position at $R^{\prime}$, which is determined by the positions of the nearest neighbor atoms $R_{n}$ of atom R. More specifically, $R^{\prime}=\sum_{n} C_{n} R_{n}, \sum_{n} C_{n}=1$, and at the minimum energy structure $\left\{\mathrm{R}_{0}\right\}, \mathrm{R}^{\prime}=\mathrm{R}$ (this uniquely determines $\mathrm{C}_{n}$ for an atom with 4 nearest neighbors). The same formula can be applied to surface atoms, which have only three nearest neighbors, if we treat the average position of all second-nearest neighbor atoms as an additional pseudo nearest neighbor atom for the purpose of carrying out $R^{\prime}=\sum_{n} C_{n} R_{n}$. The electrostatic energy in the DCM includes all the Coulomb interactions for all $q-q, q-q^{\prime}$ and $q^{\prime}-q^{\prime}$ pairs except the $q-q^{\prime}$ from the same atom. Thus we have:

$$
E_{D C M}=\frac{1}{2} \sum_{i \neq j}\left\{\frac{q\left(R_{i}\right) q\left(R_{j}\right)}{\left|R_{i}-R_{j}\right|}+\frac{q^{\prime}\left(R_{i}^{\prime}\right) q^{\prime}\left(R_{j}^{\prime}\right)}{\left|R_{i}^{\prime}-R_{j}^{\prime}\right|}+2 \frac{q\left(R_{i}\right) q^{\prime}\left(R_{j}^{\prime}\right)}{\left|R_{i}-R_{j}^{\prime}\right|}\right\}
$$

For periodic systems, this summation can be calculated via Ewald summation techniques. After making sure each ion has the correct Born Effective Charge, this model can independently determine the electrostatic charge for each ion. In this case, we will set all electrostatic charges to be zero so that the ground state of the full model is the same as that for the VFF model without charge. The details of the 
DCM were described in Ref. 30. The DCM is the most expensive part of the phonon spectrum calculations. However, as just described, its atomic charges can be predetermined based on the bulk Born charge. Thus a good practice is to calculate the DCM contribution to the dynamical matrix separately from everything else using a highly-parallelized Ewald summation routine. The result of the DCM is also subtracted from the ab initio data, with the remainder fitted by the VFF potential formula of Eq. (1).

\section{Fitting}

The fitting procedure developed for this model involves several steps. As described in the introduction, one of the main concerns is that we fit data which does not represent enough of the model's variational space that we wind up overfitting just one aspect of it. For the sake of simplicity, we will focus on just one material here, namely cadmium selenide. The ab-inito method we will use, then, is the generalized gradient approximation (GGA) of the PBE [31] density functional, with the projector augmented-wave method as implemented by the VASP code [32]. From the outset there are three types of ab initio data: the total energy, the atomic forces, and the phonon spectra of small systems.

When we wish to model a system using VFF + DCM, we first use DFT to relax a prototype system with high precision settings. A sufficiently-large supercell is used so no atom is its own neighbor or second-nearest neighbor across periodic boundaries. Then an atomic displacement is made from this ground state atomic configuration to generate the data points in our VFF parameter fitting.

We apply symmetrically unique strain modes as our first set of atomic displacement. For zinc blende, only $X X$ and $X Y$ strain modes need be used since all others in the tensor are symmetrically related to one of those two strains. For the wurtzite slab in the $X-Z$ plane, on the other hand, only $X X, Z Z$, and $\mathrm{XZ}$ strain modes are necessary since the material is unconstrained in the Y-direction. To this list of strain modes we add isometric (hydrostatic) strain if the system is periodic in all directions. With this list of strain modes we first construct perturbations for the isometric strain (if applicable) by altering the lattice parameters, ranging from all three axes being $0.5 \%$ compressed to $0.5 \%$ stretched. We then similarly construct strains for the other strain modes so that they will be of approximately the same magnitude in energy differences. This strain is important since, for some simple VFF models, all the parameters can be derived from the bulk strain moduli [9].

Second, we construct random atom perturbations by randomly displacing each atom in the system. In this study we displaced each atom in a random direction by a random distance, up to $0.5 \%$ of the shortest bond length in the system.

For each fitting in this study, ten perturbations were used for each strain mode, and then random-atom-displacement perturbations were added until the total number of perturbations was 100 . These were all then simulated with DFT to determine their energies and atomic forces. Using the DCM, the long-range electronic contribution to the energy and atomic forces under our model was determined and subtracted from these DFT-derived values, resulting in the values to be fitted by the coefficients in Eq.(1). For clarity, we note here that the ground-state distances and angles used in the 
VFF potential are simply read in from the corresponding ground-state ab initio values; they are not fitted along with the energy-term coefficients.

Conceptually, this fitting was carried out by collecting the equations for each fitting quantity (linear equations of the VFF force constants), then using a least-squares method to attempt to satisfy all of the equations simultaneously, using two types of weightings on the equations. The first weighting is applied to the force-fitting. It makes the force data contribute as much to the fitting as the energy data for each perturbation. The one exception is when the forces are all nearly zero, in which case the force data doesn't contribute to avoid divisions by zero. The second weighting divides all equations associated with a perturbation by that perturbation's energy. This fitting procedure is then expressed formally by the minimization of the following expression:

$$
\begin{aligned}
T=\sum_{i=1}^{p}\left(\frac { 1 } { | E _ { D F T , i } - E _ { D C M , i } | + \varepsilon } \left\{\left(E_{D F T, i}-E_{D C M, i}-\sum_{j=1}^{a} c_{i, j} \sigma_{j}\right)^{2}\right.\right. \\
\left.\left.+\delta\left[\frac{\left(E_{D F T, i}-E_{D C M, i}\right)^{2}}{\sum_{f=1}^{3 * n}\left(F_{D F T, i, f}-F_{D C M, i, f}\right)^{2}}\right] \sum_{f=1}^{3 n}\left(F_{D F T, i, f}-F_{D C M, i, f}-\sum_{j=1}^{a} d_{i, f, j} \sigma_{j}\right)^{2}\right\}\right)
\end{aligned}
$$

Eq.(3)

where $p$ is the number of perturbations being fitted, $E_{D F T, i}$ is the DFT energy above ground state for the $i$ th perturbation, $E_{D C M, i}$ is the DCM energy for the $i$ th perturbation, $\varepsilon$ is an approximate upper bound for the numerical variation in the DFT energy, $a$ is the number of parameters being fitted (this includes all of the $\alpha, \beta$, etc. in Eq.(1)), $\sigma_{j}$ is the $j$ th parameter (the $\alpha, \beta$, etc. in Eq.(1)), $c_{i, j}$ is the coefficient of $\sigma_{j}$ under the VFF potential's energy expression in Eq.(1) for the $i$ th perturbation, $F_{D F T, i, f}$ is the DFT force for the $f$ th force component of the $i$ th perturbation, $F_{D C M, i, f}$ is the DCM force for the $f$ th force component of the $i$ th perturbation, and $d_{i, f, j}$ is the coefficient of $\sigma_{j}$ under the VFF potential's force expression for the $f$ th force component of the $i$ th perturbation. We use $\delta$ merely to note that, should the denominator of the enclosed expression be zero (or close enough as dictated by machine accuracy), then the enclosed expression is instead replaced by zero (which means that term is not used). Examples of perturbations that necessitate this replacement are bulk strains which don't result in forces on any atoms. We note for the sake of mathematical clarity that the above expression can be rewritten in the following form:

$$
T=\sum_{i=1}^{p(3 n+1)}\left(G_{i}-\sum_{j=1}^{a} k_{i, j} \sigma_{j}\right)^{2}
$$

Eq.(4)

from which it is clear that this is a straight-forward linear least-squares problem.

After this first fitting is performed, a phonon dispersion relation can be calculated. With the DCM component of the dynamical matrix already obtained, only the VFF component is calculated, and 
the phonon modes are acquired by diagonalizing this total dynamical matrix at each k-point. This first attempt, though, tends not to be extremely accurate, so two more types of perturbations will be used in this work. The first is to use the phonon eigenmodes of this initial VFF+DCM model as atomic displacement perturbations, which can then be added to the above fitting procedure in the same way as the random displacement perturbations. The second is to use phonon modes determined by DFT itself as perturbations. Here, for each phonon mode, it is not necessary to carry out a DFT calculation. Instead, the DFT forces and energies can be determined from the phonon eigenvectors and eigenvalues. In practice, the first of these was enough to dramatically improve the phonon spectra, while the DFT phonon modes provided insignificant additional benefit.

When transferring a VFF potential to a new system, each term for the new system that directly involves an atom with new bonding topology needs to be refitted. Terms with unchanged bonding topology simply adopt the previous values. Figure 2 shows a cartoon illustrating this process for the example of adopting a bulk wurtzite VFF potential into a slab wurtzite system. These adopted values are then explicitly put into the fitting equations and their corresponding terms subtracted out, similar to how the DCM energy was subtracted from the original values. In the case of a nanowire adopting the values of a slab, there exist terms in the VFF potential that can correspond to two different terms in the slab's VFF potential. These cases are handled by simply averaging the two terms.

For more detail about the fitting and phonon spectrum calculation procedures used to produce the results in this study, see Appendix A.

Results: The results of this fitting procedure, depending on the types of perturbations included in the fitting, are shown in Figure 3 for the bulk WZ system and are shown in Figure 4 for the slab system. It may be intuitive to think that one simply needs to fit to all of the phonon eigenmodes of the DFT results in order to obtain a reasonable approximation of the mechanical behavior and phonon spectrum of a system. But as can be seen in the first row of each of these figures, this couldn't be further from the truth, and in fact one gets much better results simply by fitting bulk strains with some additional random atomic perturbations (shown in the second row). As can be seen from comparing rows three and five, inclusion of the DFT eigenstates provides essentially no additional advantage over fitting without them. The poor fitting produced from fitting just to DFT eigenstates can be visualized by plotting the dotproduct between the DFT phonon modes and the potential's phonon modes, as we've done in Figure 5. We note that the reason for this counterintuitive misfit is that, when fitted in this way, we are not directly fitting the full dynamical matrix. In a way we are only fitting the diagonal part of this dynamical matrix in the eigen-phonon-mode representation, but do not ensure that the off-diagonal part is zero. As a result, the fitted dynamical matrix is not diagonal in this representation, which results in completely different phonon modes.

As can be seen from Figures 3 and 4, it is absolutely necessary to include forces in one's general fitting procedure just to ensure no erroneous imaginary modes result. Further, from analyzing the accuracy of the acoustic modes in these same figures, it is clear that it is necessary to include the bulkstrain, random-displacement, and resultant-eigenmode perturbations in the fitting. Finally, the DFT- 
eigenmode perturbations provide little additional accuracy for the energies and forces of the model. For practical purposes, the DFT modes do not need to be included.

To demonstrate the parameter sensitivity of this fitting procedure, 100 different fittings were carried out with it for the CdSe wurtzite slab, each based on a randomly-selected half of the perturbation set described above. Statistics about the fitted parameters are summarized in Table 1. It is notable that some of the parameters varied greatly in individual fittings, especially when compared to the magnitude of the smaller parameters. Nevertheless, these different parameter sets all result in good potentials. Rated by an RMS percentage error of the energy fitting, fitting with the entire dataset resulted in a 7.6\% error for the model's ability to reproduce DFT energies. Across all of the fittings based on random halves of this dataset, the average error was only moderately higher at $9.4 \%$, with a standard deviation of $1.7 \%$ (the worst fit among the set had $14.6 \%$ error, the best $7.4 \%$ ). Visually, the resulting phonon spectra look qualitatively identical to that produced when fitting to the entire dataset. These parameter sensitivities along with good quality of the resulting potentials indicate that, to some degree, these parameters are not completely independent, they are correlated. Nevertheless, from the application point of view, this is fine since we are only concerned about the quality of the final potential.

To answer the question of how many data points are needed to obtain a good-quality potential, we have carried out different fits using different numbers of data points, and measured the quality of the resulting potentials by their RMS energy errors compared with DFT results. For each number of perturbation data points, 100 fittings each with a randomly-selected data set of the given size was carried out, and the resulting potential RMS error calculated. The results for bulk WZ and the slab system are shown in Figure 6 . As can be seen, the fitting procedure converges reasonably well within about 100 fitted-to perturbations for the bulk system, and 350 for the slab system. Since the bulk system is fitting 21 parameters at once and the slab system is fitting 53 parameters at once, the ratio of necessary fitted-to perturbations to parameters being fitted is in the range of five to seven.

To demonstrate the transferability and utility of these techniques, we have applied this VFF+DCM potential, fitted to a CdSe slab, to a sequence of wurtzite CdSe nanowires ranging in diameter from $26 \AA$ to $52 \AA$, each eight times the unit-cell length along its c-axis. The DCM component of the dynamical matrix costs most of the computation time. For the 864-atom wire the computation took about 49 seconds on 648 cores, for the 1538-atom wire it took about 2 minutes and 30 seconds on 1152 cores, for the 2400 -atom wire it took about 4 minutes 21 seconds on 1800 cores, and for the 3456-atom wire it took about 9 minutes 17 seconds on 2592 cores, with all calculations being conducted on NERSC's Hopper. The resulting phonon dispersion relations and densities of states are plotted in Figure 7 . We see that, from the small nanowire to the large one, the density of states approaches the bulk values. However, there are a few consistent surface modes. In particular, the highest-energy phonon mode comes from the corner, with the $\mathrm{Cd}$ and Se atoms there stretching out away from their corners and each other. There is also a branch of acoustic phonon modes with large amplitude from the surface. This corresponds to the twisting of the wire.

One can also relate the phonon spectrum of the nanowire in Fig.7 to the bulk dispersion in Fig.1(c). It can be clearly seen that some of the bulk band structure lines from Fig.1(c) are in Fig.7. In particular the 
entire phonon dispersion curves of the r-A panel of Fig.1(c) can be seen in Fig.7, as these are the dispersion curves along the wire axis. There are different ways to describe the confinement effects of the phonon modes in nanostructures. In a k-point folding approach [33], one can attempt to identify the nanostructure phonon modes by their corresponding modes and k-points in the bulk dispersion relationship. But the exact determining of the k-point value is not trivial due to the cylindrical shape of the nanowire phonon mode envelope functions, and possible-different boundary conditions at the surface. The phonon confinement model (PCM) of Richter, Campell and Fauchet $[34,35]$ is often used to describe the Raman spectrum shape and peak shift of nanostructures using the bulk phonon dispersion relation. In the PCM model, a Gaussian like envelop function of the nanostructure phonon mode is constructed by many bulk phonon modes through a weight function in k-space. Such weight function can change the shape and peak position of the Raman spectrum. It will be interesting in the future, to use the nanowire phonon modes explicitly calculated in our method to check the PCM model for nanowire systems [36].

\section{Conclusion}

Using the model described in this study and the fitting procedure laid out, it was possible to accurately reproduce the ab initio phonon spectra for both bulk and slab CdSe systems, with only a few phonon modes which slightly differ from the ab initio results. Most of these modes are well known to be difficult to fit very precisely for classical force-field models, specifically the transverse acoustic modes away from the gamma point $[29,37]$. The average energy error for phonon modes in these fitted spectra is approximately $7 \%$, attributed predominantly to the few poorly-fitted phonon modes.

The best fitting procedure we found is to fit the parameters in stages, while transferring the previous fitted parameters into more complex systems. In our case, we should first fit the bulk CdSe systems. After that, these parameters will be fixed and transfer into the slab fitting with new parameters introduced related to surface atoms of the slab.

All the fitting data points are formulated as strains/displacements from the ground state configuration, for their energies and atomic forces. We found that it was necessary to fit both energy and force data from ab initio calculations in order to produce a well-fitted phonon spectrum. It is useful to follow a two-step process. First use purely random perturbations to produce the fitting data, then after the first fitting with a first-round VFF parameter set, generate a set of VFF phonon modes, then use these phonon modes as perturbations to generate another set of ab initio fitting data. One can also use ab initio phonon modes as the perturbations, but we found that they only provide rather small benefits. Thus, in practice, it is not necessarily to include them. We also found that only including the phonon modes (no random displacement) as the perturbation will not produce good phonon modes.

It is important to adjust the weights for each fitted value, so an optimized result will be obtained. This is particularly true for a balance between the energy term and the force term. It was found that the best results were achieved when the force term in a fitted-to perturbation contributes as much as its energy term of that perturbation in the task function. This provides a way to adjust the relative weights between energy and the force terms. Between different perturbations, it is found that 
the best result is obtained when each perturbation contributes to the task function in proportion to its energy (not energy square, and not independent of energy either). Thus a weight factor proportional to the inverse of the fitted energy should be used (Eq.(3)). This modification is necessary in order to include the influence of the low-energy modes. Conversely, fitting with all perturbations with equal weight will result in big errors in optical modes without substantial improvements to the rest of the spectrum.

Through different fitting tests, it was found that the number of ab initio perturbations necessary to fit a given number of parameters (for the type of VFF model chosen) was on the order of five to seven perturbations per parameter being fitted (note, each perturbation will generate up to $3 \mathrm{~N}+1$ ab initio data points including both energy and atomic force values). Beyond this number of perturbations, the fitting quality will be saturated. Thus the remaining inaccuracy will be from the model itself, not from the fitting procedure. Thus to have a more accurate result, a better model will need to be chosen.

Finally, through analyzing the specific potential parameters that resulted from fitting to different perturbation sets, it was found that the individual potential parameters could vary significantly in value from fitting to fitting. Nevertheless, each resulting potential produces a quantitatively-good phonon spectrum, with little difference from each other. From this we can conclude that there is some degree of parameter interdependence and correlation. As a result, the parameters cannot be uniquely determined. Nevertheless, it is not necessary to sort out a unique set of independent parameters, since all the fitted potentials work well in terms of producing high quality phonon spectra.

\section{Appendix A}

A step by step wurtzite bulk fitting and phonon spectrum calculation procedure: (1) To apply the described procedure for fitting a VFF potential to a CdSe wurtzite bulk system as simulated by DFT, a rhombohedral supercell containing 16 atoms (the minimum unit cell doubled in the $a$ - and $b$ - directions) is first constructed and fully relaxed for its internal atomic positions as well as the unit cell parameters. The result of this relaxation has cell parameters of $4.388 \AA$ and $7.160 \AA$ along the a- and c-axes respectively, with the bond length parallel to the c-axis $2.691 \AA$ and the other bonds $2.685 \AA$ in length. This is the smallest supercell where no atom is its own third-nearest neighbor, which makes the application of the VFF model simple and effective.

(2) A symmetry operation checking program is used to determine which atoms are symmetrically related to each other in the supercell used above. For the wurtzite bulk, it simply finds that all of the cadmium atoms are symmetric to each other, as well as are the selenium atoms. Similarly the routine also determines the symmetry relationships between all the bonds, bond angles, and bondangle pairs in the structure. For the wurtzite bulk structure, the list of symmetrically-unique features includes two atoms, two bonds, four bond angles, and seven bond-angle pairs. In our VFF model, there are parameters that correspond to each of these features.

(3) Using the bonding topology data just obtained from the atomic geometry, the coefficients $C_{n}$ in the DCM model used to determine the position $\mathrm{R}^{\prime}$ of the second charge $\mathrm{q}^{\prime}$ are calculated. More specifically, this is calculated for each atom by solving $R^{\prime}=\sum_{n} C_{n} R_{n}$ and $\sum_{n} C_{n}=1$ for $\mathrm{n}$ running over 
the four nearest neighbors, and at the equilibrium configuration, $R^{\prime}$ is the same as $R$ (the atom considered). For our wurtzite $\mathrm{CdSe}$, we obtained $\mathrm{C}_{n}=0.24845$ for the atom bonded to in the c-direction, and $C_{n}=0.25052$ for the other three nearest neighbor atoms. In the subsequent fitting, these $C_{n}$ coefficients are kept fixed.

(4) One can treat the second charge $q^{\prime}$ at $R^{\prime}$ as another atomic charge only with the exception that it doesn't interact with $q$ at R. Thus, an Ewald summation program can be used to calculate the DCM electrostatic energy, and its second derivatives relative to $\mathrm{R}$ and $\mathrm{R}^{\prime}$. The equation $R^{\prime}=\sum_{n} C_{n} R_{n}$ can be used to further convert the $\mathrm{R}^{\prime}$ derivative to $\mathrm{R}$ derivative, which will yield the dynamical matrix relative to $R$ for the electrostatic energy in the DCM model. Numerically, the second derivative is obtained by displacing one atom, then calculating the atomic force changes in all the other atoms. It is noteworthy that only the symmetrically distinct atoms need to be displaced. This, combined with the fact that, to get the change in atomic force for each atom, one only needs to consider the chargeCoulomb terms related to the displaced atom (all other atoms with undisplaced second charges have their charges cancel), allows for substantial reduction of the computation. Specifically, the computational cost per row in the dynamic matrix scales as $\mathrm{O}(\mathrm{N})$ where $\mathrm{N}$ is the number of atoms, and the cost for the entire matrix then scales as $\mathrm{O}\left(\mathrm{N}^{2}\right)$. We used a supercell to calculate the dynamic matrix and the bulk phonon spectrum. This supercell has a size quadrupled in the a- and b- directions and doubled in the c-direction, containing 128 atoms. However, due to symmetry, we only need to calculate the rows of the matrix that correspond to the four atoms in the primary cell (e.g., displacing only these four atoms, or even only two atoms in the primary cell taking advantage of the symmetry between the two Cd and Se atoms).

(5) The parameters $q^{\prime}$ in the DCM model are not fitted. They are taken from the Born effective charges for the system. Our fitting is only concerning the VFF bond components. First we produce perturbations in the supercell used, and simulate them with DFT to produce energy and force data. The perturbations for the wurtzite bulk system include bulk strains and random atomic displacements. The bulk strains include hydrostatic strains up to $0.5 \%$, a-axis strains up to $1.5 \%$, c-axis strains up to $1.5 \%$, ab-axes shear strains up to $2.5 \%$, and a-c-axes shear strains up to $2.5 \%$. Each of these strain modes was calculated in eleven increments from $-100 \%$ to $100 \%$ of the maximum (e.g. the hydrostatic strains simulated were $-0.5 \%,-0.4 \%,-0.3 \%,-0.2 \%,-0.1 \%, 0 \%, 0.1 \%, 0.2 \%, 0.3 \%, 0.4 \%$, and $0.5 \%)$. The random atomic displacements were produced by generating three random numbers in the range of $-0.00775 \AA$ to $0.00775 \AA$ for each atom and using them to determine its $x, y$, and $z$ displacements (the largest displacement possible is $0.5 \%$ of the shortest bond length of the system; all atoms are perturbed at the same time). In total, we have 50 random displacement perturbations and 50 strain perturbations.

(6) After all of these perturbations are calculated with DFT, the Ewald summation technique (discussed above) is used to calculate the DCM charge contribution to the energies and forces of all these perturbations. With the DFT data and the DCM contributions we can now fit our VFF potential parameters via the minimization of the right-hand side of Equation 3 . All that is needed are the $c_{i, j}$ and the $d_{i, f, j}$ of Equation 3, which are computed by applying the potential formalism of Equation 1 to each perturbation for the $c_{i, j}$ and the formalism for the force of this potential for the $d_{i, f, j}$. The one question 
left, then, is what value should be used for $\varepsilon$ in Equation 3. The primary purpose of this constant is to account for errors inherent to the numerical methods used in DFT calculations. We want $\varepsilon$ to be large enough so that the fitting will not be dominated by perturbations with small (and possible inaccurate) energy increases from the ground state. In this work we have used $1 \mathrm{meV}$ for $\varepsilon$.

(7) There is an issue which became apparent after fitting a VFF potential to the data set: the $\beta$ values vary greatly when compared to the other terms as the fitted-to data set changes. This is a result of the symmetry of the system which does not allow for independent fitting of all $\beta$ terms under the current procedure. Specifically, for binary bulk systems (including both zinc-blende and wurtzite) in which swapping the two atom types results in the same system, this fitting procedure only fits the sum of two $\beta$ terms which are symmetric to each other (completely correlated). Thus, without the loss of generality, we have restricted the corresponding two $\beta$ coefficients to be the same (between Se-Cd-Se and $\mathrm{Cd}$-Se-Cd angles). This has fixed the $\beta$ variation problem. After this, for the bulk wurtzite structure, we have two $\alpha$, two $\beta$, six $\gamma$, four $\delta$, and seven $\varphi$ terms to be independently fitted.

(8) With the VFF parameters in hand we can now calculate the VFF contribution to the dynamical matrix. This is accomplished in a similar fashion to the procedure used for the DCM contribution. There is one final contribution to the dynamical matrix called the "nonanalytic term" that is due to the long-range-limit effects of Born effective charge. This only happens for systems that are infinitely periodic in all three dimensions, and it cannot be captured by either DFT calculation, or our supercell Ewald-energy calculation [38]. Physically, the nonanalytic component corresponds to the effect of the macroscopic electric field produced by the macroscopic polarization in long-wavelength phonon modes, and thus results in the energy splitting between longitudinal and transverse optical mode at the Gamma point. This nonanalytic (singular at the Gamma point) term has to be added on top of our supercell-calculated dynamic matrix in order to obtain a phonon band structure, which then can be compared with experiments.

(9) After this initial fitting is completed, the result might not be as accurate as desired (as illustrated by Figure 3). To improve the fitting further, we take the gamma-point phonon eigenvectors that resulted from the fitted VFF Hamiltonian and use them as perturbations to generate DFT energy and force data, then add them to fitting (following the same procedure as described above). The final resulting VFF Hamiltonian and the phonon spectrum are found to be of high quality.

A step by step procedure for wurtzite slab fitting and phonon spectrum calculation: (1) The procedure for fitting a VFF potential to a CdSe wurtzite slab, as well as calculating its phonon spectrum, closely follows that used for the wurtzite bulk, with changes made to account for differences in topology and for the adoption of the bulk parameters in the slab interior.

(2) In this study, the wurtzite slab constructed has (10-10) surfaces in a rectangular unit cell with the $y$-axis normal to these surfaces and the $z$-axis parallel to the c-axis of the bulk. The slab used is 13.25 $\AA$ thick with $63.55 \AA$ of vacuum above, the lateral dimensions of the slab are $8.68 \AA$ and $14.33 \AA$, while the $13.25 \AA$ thickness corresponds to four layers of bulk unit cells. The supercell contains 64 atoms while the minimum unit cell contains 16 . This slab was fully relaxed using DFT. The same symmetry-detecting 
routine is used to detect the symmetry and distinct features of this system. The unique features for this slab system include 8 atoms, 11 bonds, 28 bond angles, and 62 bond-angle pairs.

(3) The $C_{n}$ coefficients for the slab are calculated in the same way as for the bulk. However, for each atom at the surface of the slab, there are only three nearest-neighbor atoms, which make it impossible to satisfy $R^{\prime}=\sum_{n} C_{n} R_{n}$ and $\sum_{n} C_{n}=1$ if only the nearest neighbor atoms are used for $\mathrm{n}$. As a result, we have used the average position of that atom's second-nearest neighbors as the fourth position $R_{n}$ in this equation. Furthermore, while the bulk values of the charges $q$ and $q^{\prime}$ can be determined by a simple symmetry argument and the atomic Born effective charge, no such argument exists for the atoms at the surface of the slab. One can attempt to use DFT calculation to determine the Born effective charge tensors of the surface atoms, and then use those to approximate a $q$ and $q^{\prime}$. However, to avoid such calculations and complications, we have simply adopted the bulk charge values $q$ and $q^{\prime}$ for all atoms except the two three-bonded surface atoms. For these two surface atoms (denoted as 1 and 2), we have set $q=-q^{\prime}$, as well as $q(1)=-q(2)$. The remaining free parameter $q(1)$ is determined by requiring that the Born effective charges of the other atoms are as close to their bulk values as possible. This however does not guarantee the surface atoms have the correct Born effective charge. The local effects of not having accurate Born effective charges at the surface will likely be absorbed by the fitting of the bonding terms in the VFF model there.

(4) With the values of the charges $q$ and q' fixed, as well as the way to calculate $R^{\prime}$ for surface atoms determined, we can now calculate the DCM contribution of the dynamical matrix in the same way as for the bulk, keeping in mind that this DCM model might involve the second nearest neighbors for the surface atoms. For these surface atoms, they have three nearest neighbors and eight second-nearest neighbors. We now construct perturbations of the slab system in the same way as for the bulk, except we only consider a subset of the strain modes. Specifically, we consider $x$-axis strains up to $1.5 \%$, z-axis strains up to $1.5 \%$, and $x-z$-axes shear strains up to $2.5 \%$. In total, we have 30 strain perturbations, and 70 random atom-displacement perturbations.

(5) After calculating the DFT and DCM energies and forces of our perturbations, we can fit a VFF potential to our slab following Equation 3, as we did before for bulk. The previously fitted bulk coefficients are used for all of the VFF potential terms except those directly involving the surface atoms (three-bonded atoms). With all but the surface's VFF terms predetermined, we can plug these terms into Equation 3 and fit for the surface-related VFF coefficients. Because the relaxation of the slab results in a buckling of the surface bonds, the symmetry between the $\beta$ terms that existed in the bulk no longer exists. As a result, there are $3 \alpha, 10 \beta, 16 \gamma, 10 \delta$, and $14 \varphi$ coefficients to be fitted for the wurtzite (1010) surface.

(6) After this initial fitting, we can calculate the total VFF dynamical matrix and the corresponding phonon spectrum for the slab system. And as was done for the bulk procedure at this stage, the gamma-point phonon eigenvectors of this first fitting can be used to construct additional atomic perturbations, followed up by DFT calculations, and the resulting data can be incorporated into a second fitting to achieve better accuracy. 
Acknowledgment: This work was supported through the Theory of Material project by the Director, Office of Science (SC), Basic Energy Science (BES)/Materials Science and Engineering Division (MSED) of the U.S. Department of Energy (DOE) under the contract No. DE-AC02-05CH11231. This work uses the resources of National Energy Research Scientific Computing center which is funded by U.S. Department of Energy.

\section{References}

[1] C. A. Coulomb, Histoire de l'Académie Royale des Sciences 569 (1788)

[2] J. E. Lennard-Jones, Proc. R. Soc. Lond. A 106, 463 (1924)

[3] P. M. Morse, Phys. Rev. 34, 57 (1929)

[4] R. Eisenschitz and F. London, Zeitschrift für Physik 60, 491 (1930)

[5] R. A. Buckingham, Proc. R. Soc. Lond. A 168, 234 (1938)

[6] F. Carlsson, P. Linse, M. Malmsten, J. Phys. Chem. B 105, 9040 (2001)

[7] F. Carlsson, M. Malmsten, P. Linse, J. Phys. Chem. B 105, 12189 (2001)

[8] C. Pryor; J. Kim; L.W. Wang; A. Williamson; A. Zunger, J. Appl. Phys. 83, 2548 (1998)

[9] J. Tersoff, Phys. Rev. B 37, 6991 (1988)

[10] M. S. Daw and M. I. Baskes, Phys. Rev. B 29, 6443 (1984)

[11] P. N. Keating, Phys. Rev. 145, 637 (1966)

[12] N. L. Allinger, J. Am. Chem. Soc. 99, 8127 (1977)

[13] T. Schauman, W. Braun, and K. Wutrich, Biopolymers 29, 679 (1990)

[14] A. Warshel and S. Lifson, J. Chem. Phys. 53, 582 (1970)

[15] W. D. Cornell, et al., J. Am. Chem. Soc. 117, 5179 (1995)

[16] B. R. Brooks, et al., J. Comp. Chem. 4, 187 (1983)

[17] M. Mollhoff and U. Sternberg, J. Mol. Model. 7, 90 (2001)

[18] S. J. Weiner, et al., J. Amer. Chem. Soc. 106, 765 (1984)

[19] A.C.T. van Duin, A. Strachan, S. Stewman, Q. Zhang, X. Xu, W.A. Goddard,III. J. Phys. Chem. A 107, 3803 (2003)

[20] H. Sun, J. Phys. Chem. B 102, 7338 (1998)

[21] T. A. Halgren, J. Comp. Chem. 17, 490 (1996)

[22] G. Csanyi, T. Albaret, M.C. Payne, A. De Vita, Phys. Rev. Lett. 93, 175503 (2004)

[23] D.B. Laks, L.G. Ferreira, S. Froyen, A. Zunger, Phys. Rev. B 46, 12587 (1992)

[24] F. Zhou, W. Nielson, Y. Xia, V. Ozolins, Phys. Rev. Lett. 113, 185501 (2014)

[25] Y. Luo, L.W. Wang, ACS Nano. 4, 91 (2010)

[26] H. L. McMurray, A. W. Solbrig, J. K. Boyter, and C. Noble, J. Phys. Chem. Solids 28, 2359

[27] R. Tubino, L. Piseri, and G. Zerbi, J. Chem. Phys. 56, 1022 (1972)

[28] Z. Sui and I. P. Herman, Phys. Rev. B 48, 17938 (1993) 
[29] A. Paul, M. Luisier, and G. Klimeck, J. Comput. Electron. 9, 160 (2010)

[30] C. Barrett, L.W. Wang, Phys. Rev. B, 91, 235407 (2015)

[31] J.P. Perdew, K. Burke, and M. Ernzerhof, Phys. Rev. Lett. 77, 3865 (1996)

[32] G. Kresse and J. Furthmüller, Phys. Rev. B 54, 11169 (1996)

[33] C.Y. Yeh, S.B. Zhang, A. Zunger, Phys. Rev. B 50, 14405 (1994).

[34] H. Richter, Z.P. Wang, L. Ley, Solid State Commun. 39, 625 (1981).

[35] I.H. Campell, P.M. Fauchet, Solid State. Commun. 58, 739 (1986).

[36] N. Begum, A. S. Bhatti, F. Jabeen, S. Rubini, F. Martelli, pp.414, "Nanowires", edited by Paola Prete (March 2010, INTECH, Croatia).

[37] R. M. Martin, Phys. Rev. 186, 871 (1969)

[38] X. Gonze and C. Lee, Phys. Rev. B 55, 10355 (1997)

\section{Figures}
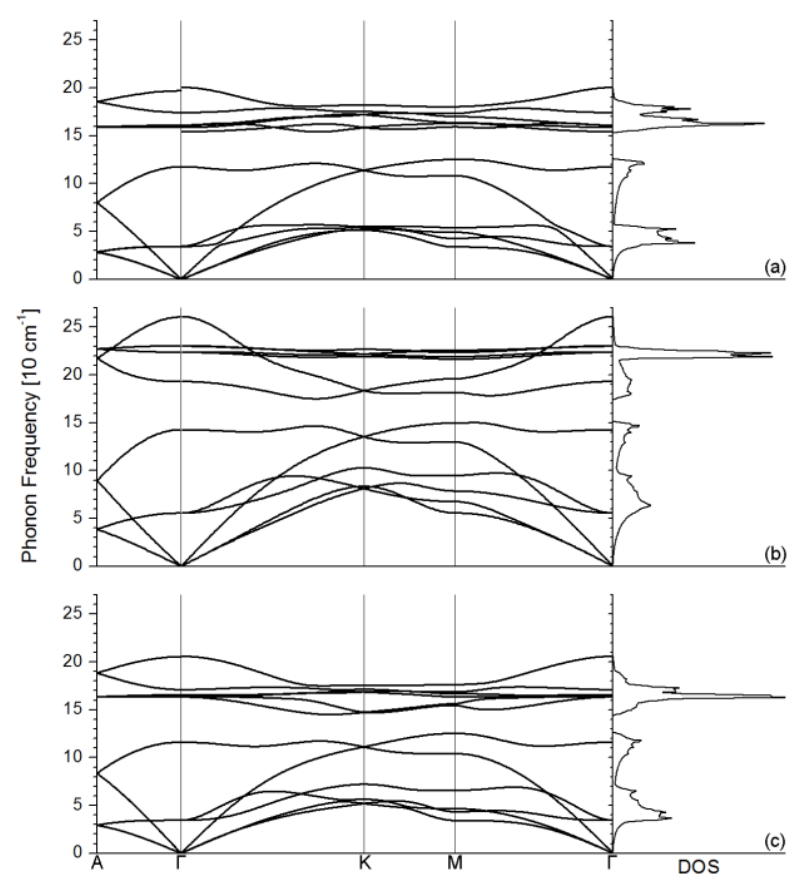

Fig. 1. Phonon dispersion relations in high-symmetry directions, with accompanying density of states plots, for bulk wurtzite CdSe. (a) VASP GGA DFT. (b) A VFF model fitted to experimental bulk strain moduli that is used for structural geometry optimization. (c) VFF+DCM, fitted according to the techniques presented here. The slight discontinuity at $\Gamma$ for some optical phonon modes results from the different splitting between those modes as $\Gamma$ is approached from different directions, due to the anisotropy of the macroscopic electric response inherent to wurtzite structures. 


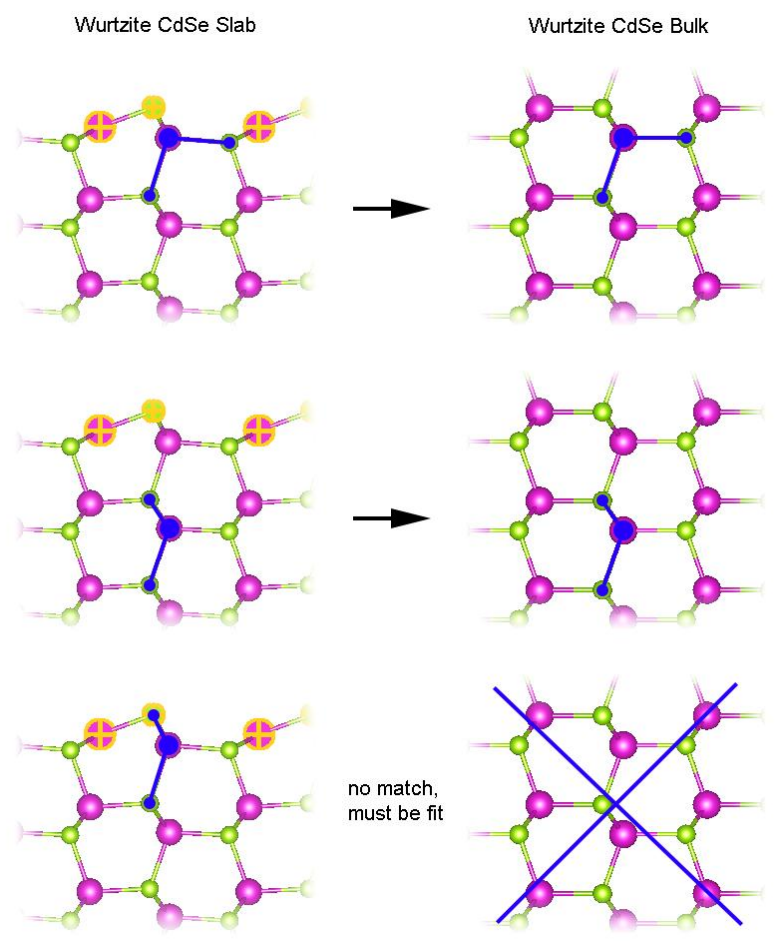

Fig. 2. In the process of applying the VFF potential of a wurtzite bulk system to a wurtzite slab, bond angles, denoted in blue, are identified in the wurtzite slab on the left and an attempt is made to find similar bond angles in the wurtzite bulk on the right. For a given pair of bond angles between the systems, if their atoms are in the same bonding topology and of the same type, their bonds are of similar lengths and orientations, and their angles are of similar measure, then they are considered to be a match. Coefficients of the VFF potential that correspond to the bond angle in the wurtzite bulk are adopted for the corresponding bond angle in the slab. Similar is true for the bond coefficients and the angle-angle coefficients of the potential. Atoms of the slab that have bonding topology not represented in the bulk are marked with yellow in the figure. Any energy term of the VFF potential involving one of those atoms will have to be refitted since they are unrepresented in the bulk structure. 

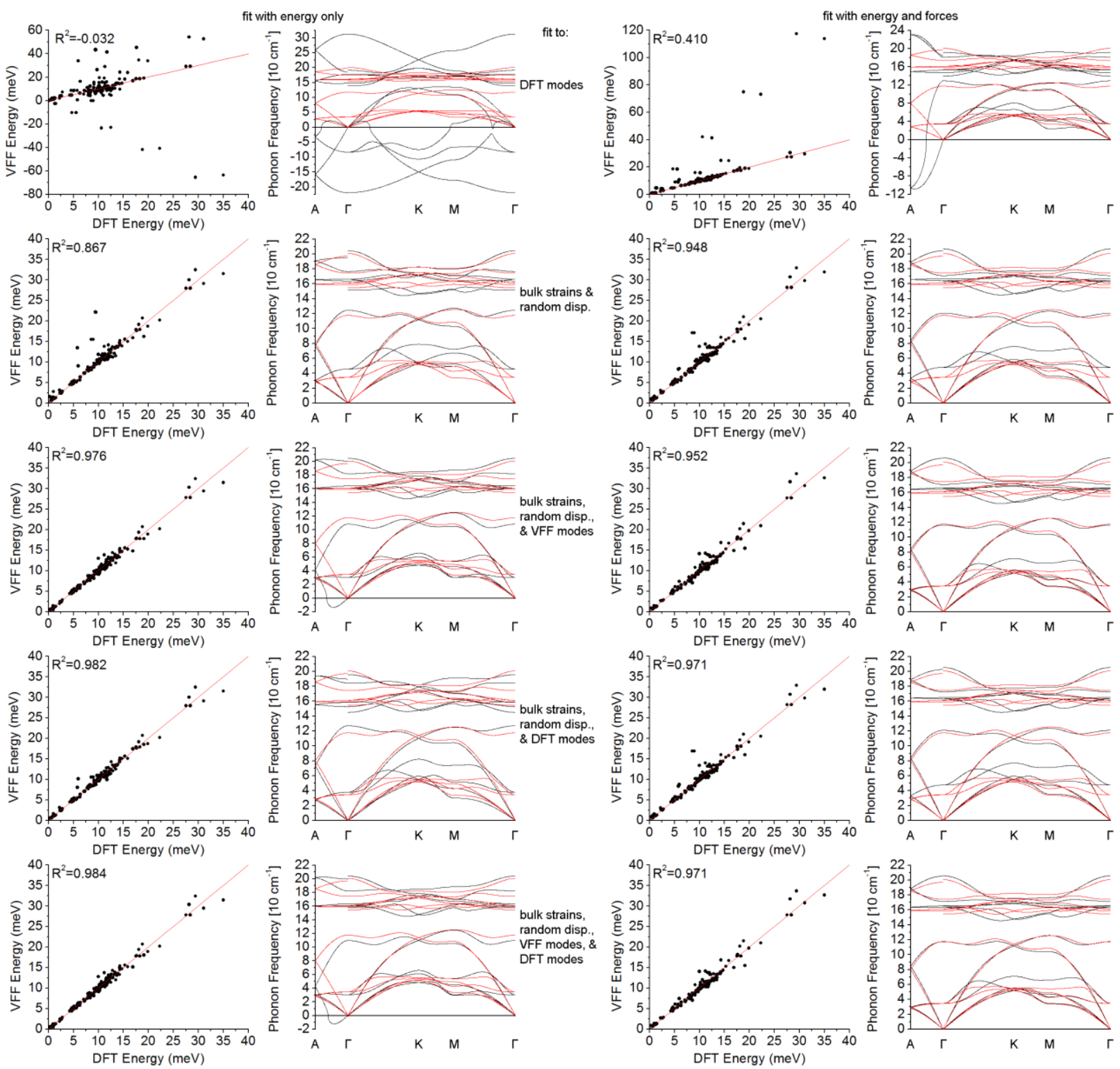

Fig. 3. Plots of the energies of all of the possible perturbations considered in the WZ fitting procedure for various modifications of the fitting procedure, and the phonon spectra that result from those fitting procedures. Plots on the left show fittings that only attempted to fit the energies, while the right shows both energy and force data being utilized. The first row shows the use of only DFT-eigenstate perturbations being used; the second bulk-strain and random-displacement perturbations only; the third bulk-strain, random-displacement, and resultant-eigenstate perturbations; the fourth bulk-strain, random-displacement, and DFT-eigenstate perturbations; and the fifth shows use of all five. The Rsquared value for the energy plots are inset. 

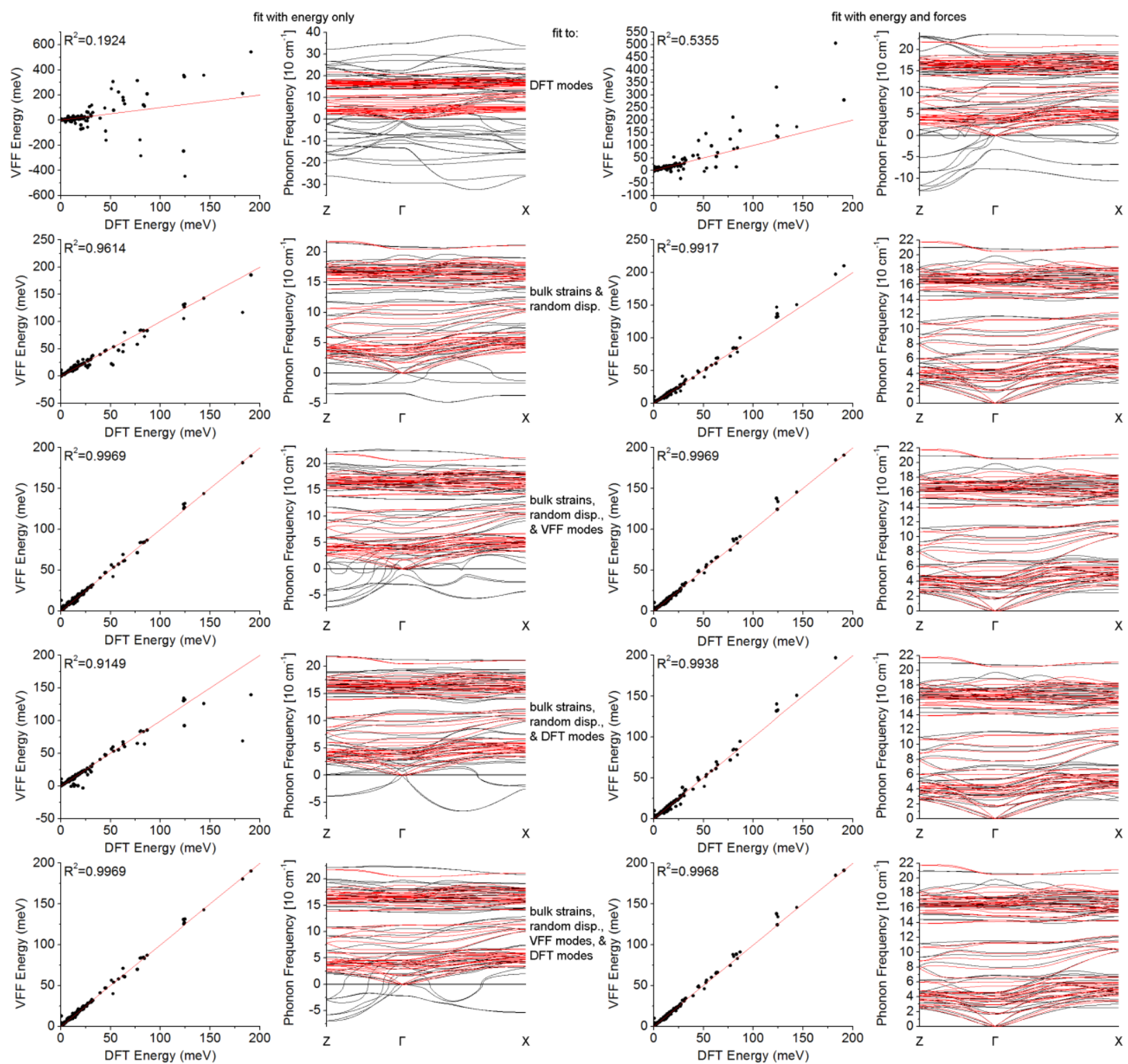

Fig. 4. Plots of the energies of all of the possible perturbations considered in the slab fitting procedure for various modifications of the fitting procedure, and the phonon spectra that result from those fitting procedures. Plots on the left show fittings that only attempted to fit the energies, while the right shows both energy and force data being utilized. The first row shows the use of only DFT-eigenstate perturbations being used; the second bulk-strain and random-displacement perturbations only; the third bulk-strain, random-displacement, and resultant-eigenstate perturbations; the fourth bulk-strain, random-displacement, and DFT-eigenstate perturbations; and the fifth shows use of all five. The Rsquared value for the energy plots are inset. 


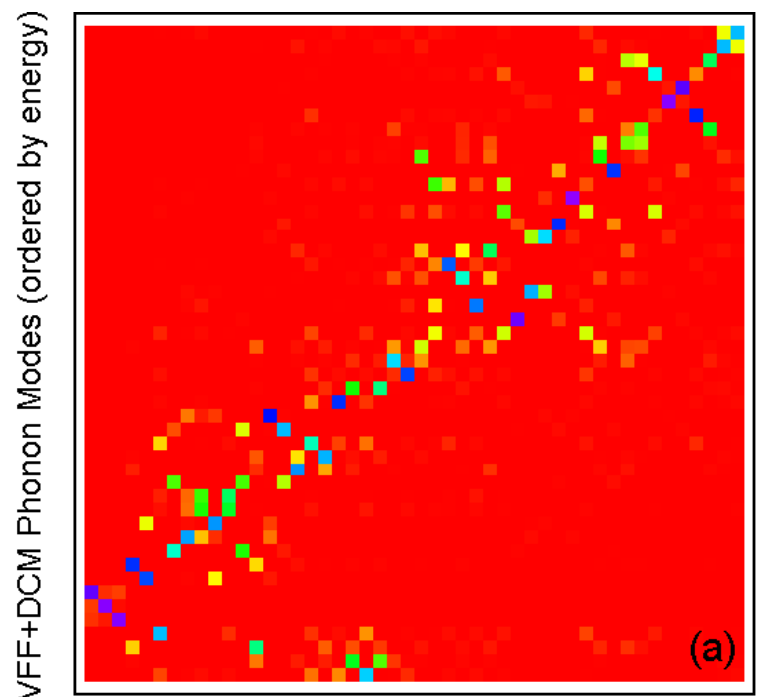

DFT Phonon Modes (ordered by energy)

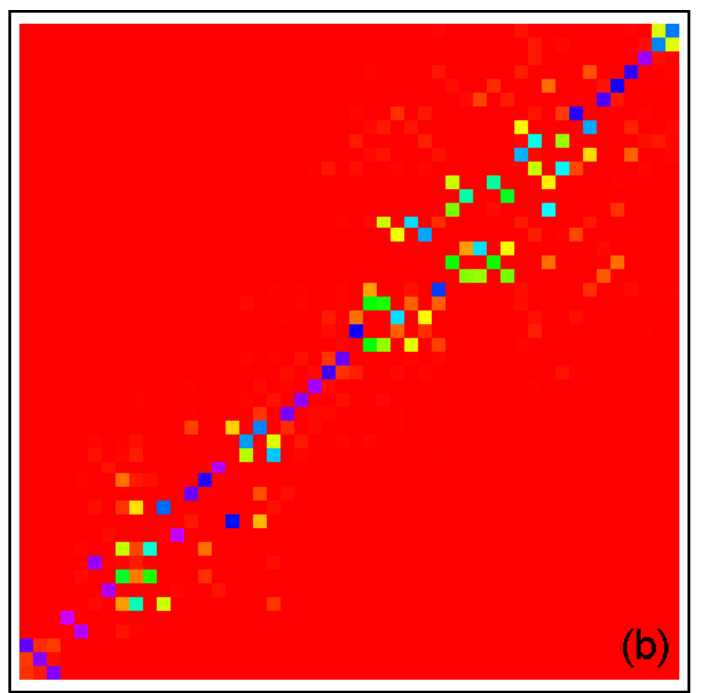

DFT Phonon Modes (ordered by energy)

Fig. 5. Plots of the dot product of the phonon modes of the CdSe slab between the DFT results and the fitted results for (a) a potential fitted only to DFT phonon eigenstates and (b) a potential fitted to all perturbations. The column is the index of the DFT phonon modes and the row is the index of the fitted potential phonon modes. Each set of phonon modes is ordered from lowest to highest energy, starting in the bottom-left. Note that five modes in (a) wind up with negative energies even though they mix heavily with modes throughout the acoustic bands, while acoustic modes appear to mix randomly among themselves, as do the optical modes, and there is even noticeable mixing between acoustic and optical modes. (b) has none of these problems. It is worth noting that degeneracies give some appearance of extra mixing, especially in (b), due to the eigenvector choice within degenerate spaces and index ordering between the two sets of phonon modes being determined by numerical artifacts.

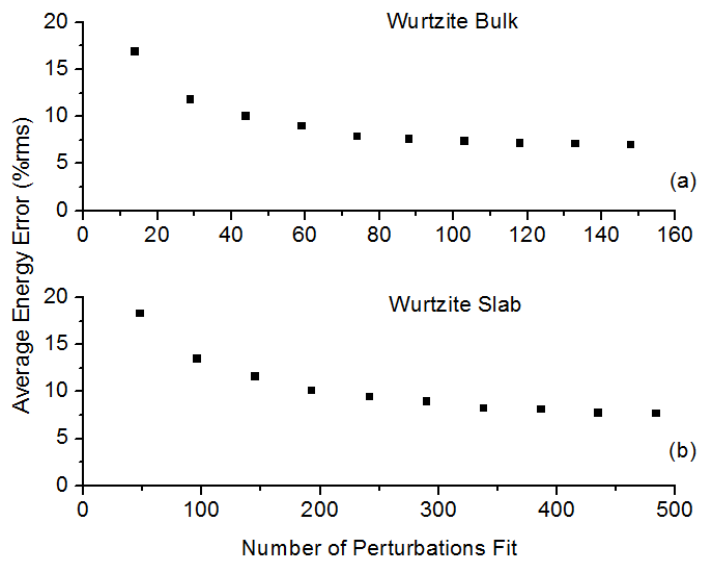

Fig. 6. Average energy-fitting errors for various numbers of fitted-to DFT perturbations for (a) a CdSe bulk and (b) a CdSe slab. Each point represents the average of the RMS percentage errors for 100 different fittings. 

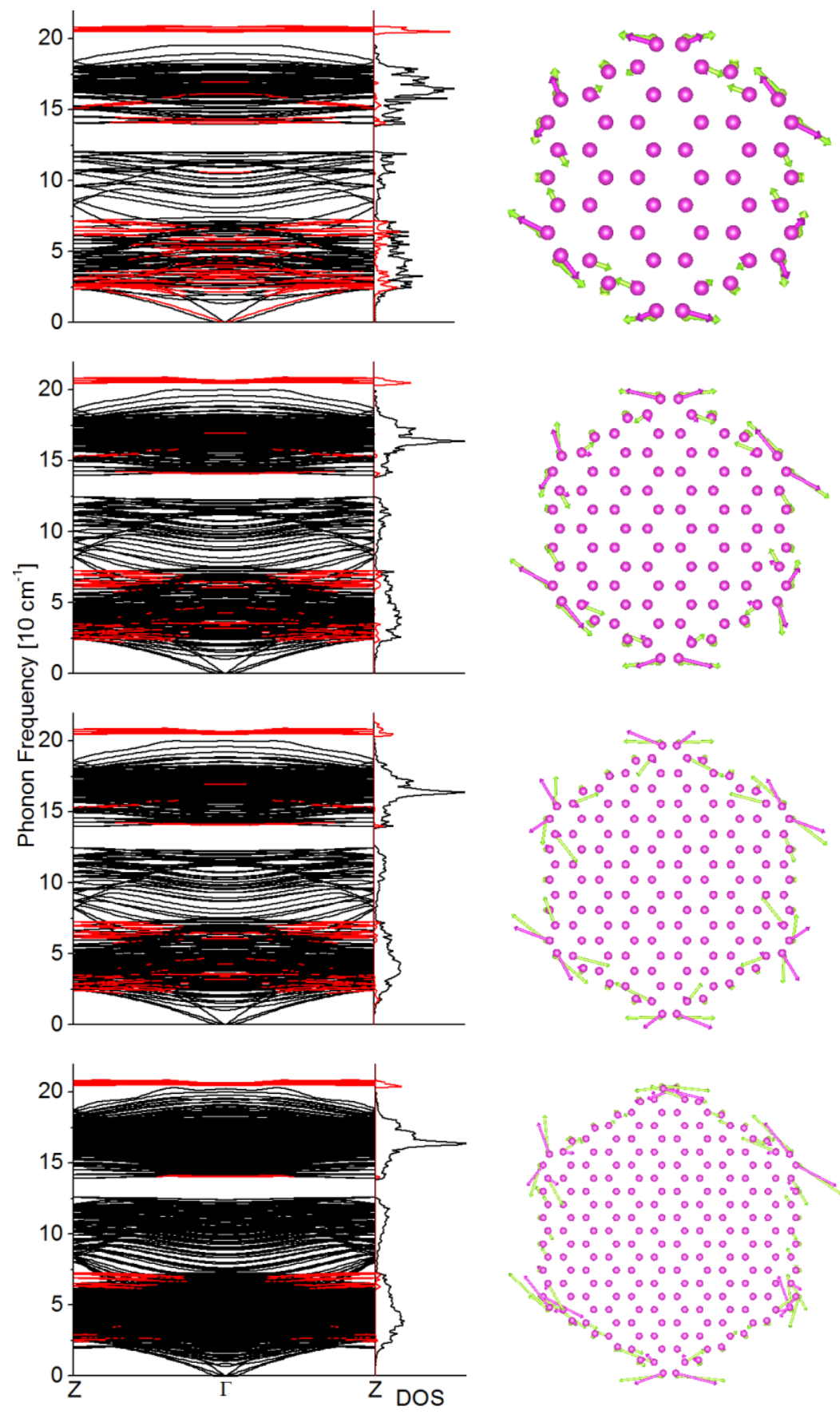

Fig. 7. From top to bottom, the phonon dispersions and density of states are plotted for the 864-, 1538-, 2400-, and 3456-atom nanowires, respectively. To the right of each is the top phonon mode at the gamma point, represented by displacement vectors, applied to each atomic structure. Purple spheres represent the $\mathrm{Cd}$ ions and the corresponding purple vectors represent their displacements in the top mode. Green spheres represent the Se ions and the corresponding green vectors represent their displacements in the top phonon mode. The views of the atomic structures are orthographic projections looking down the axes of the nanowires. The displacements for all atoms are almost entirely in the plane perpendicular to this axis. 


\begin{tabular}{|r|r|r|r|r|}
\hline & $\begin{array}{c}\text { Average } \\
(\mathrm{N} / \mathrm{m})\end{array}$ & $\begin{array}{c}\text { Average } \\
\text { Magnitude } \\
(\mathrm{N} / \mathrm{m})\end{array}$ & $\begin{array}{c}\text { Average } \\
\text { Standard } \\
\text { Deviation }\end{array}$ & SD/Mag \\
\hline$\alpha$ & 26.77 & 26.77 & 0.75 & 0.03 \\
\hline$\beta$ & 2.07 & 2.97 & 1.27 & 0.43 \\
\hline$\gamma$ & -1.28 & 3.90 & 0.87 & 0.22 \\
\hline$\delta$ & 2.98 & 3.16 & 0.65 & 0.21 \\
\hline$\phi$ & 0.34 & 1.93 & 1.13 & 0.59 \\
\hline
\end{tabular}

Table 1. Summarized statistics for 100 fittings of the VFF potential to randomized halves of the dataset used for fitting the CdSe wurtzite slab. For each different $\alpha$ term in the VFF potential, the average was computed across 100 different fittings and then the average of those averages across all such $\alpha$ terms is reported here, and similar for the average of the averages of the absolute values and the average of the standard deviations of those results for each term. This was also carried out for the $\beta, \gamma, \delta$, and $\phi$ terms, all of which is reported here. 